Acta Crystallographica Section E

Structure Reports

Online

ISSN 1600-5368

\section{Bis(isobutylammonium) phthalate monohydrate}

\section{Zakaria Norzalida, Azhar Ariffin and Seik Weng Ng*}

Department of Chemistry, University of Malaya, 50603 Kuala Lumpur, Malaysia

Correspondence e-mail: seikweng@um.edu.my

Received 14 May 2008; accepted 20 May 2008

Key indicators: single-crystal X-ray study; $T=100 \mathrm{~K}$; mean $\sigma(\mathrm{C}-\mathrm{C})=0.004 \AA$; $R$ factor $=0.062 ; w R$ factor $=0.156 ;$ data-to-parameter ratio $=18.0$.

$N$-Isobutylphthalimic acid hydrolyzes to the title salt, $2 \mathrm{C}_{4} \mathrm{H}_{12} \mathrm{~N}^{+} \cdot \mathrm{C}_{8} \mathrm{H}_{4} \mathrm{O}_{4}{ }^{-} \cdot \mathrm{H}_{2} \mathrm{O}$, which adopts a hydrogen-bonded layer structure. In the anion, the carboxylate groups are twisted with respect to the benzene ring [dihedral angles = $43.8(1)$ and $50.9(1)^{\circ}$ ].

\section{Related literature}

For kinetic studies relating to the hydrolysis of $\mathrm{N}$-isobutylphthalimic acid, see: Ariffin \& Khan (2005); Khan \& Ariffin (2003).
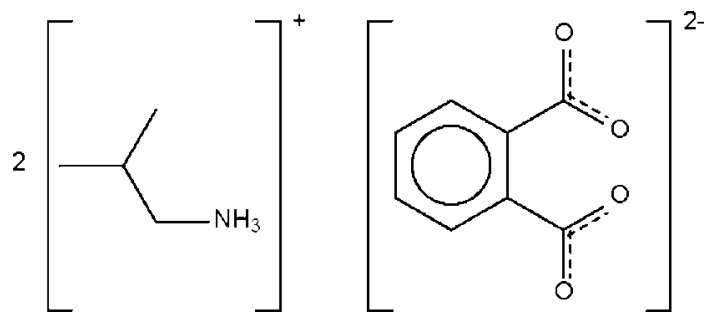

$\mathrm{H}_{2} \mathrm{O}$

\section{Experimental}

Crystal data

$2 \mathrm{C}_{4} \mathrm{H}_{12} \mathrm{~N}^{+} \cdot \mathrm{C}_{8} \mathrm{H}_{4} \mathrm{O}_{4}{ }^{2-} \cdot \mathrm{H}_{2} \mathrm{O}$

$M_{r}=330.42$

Triclinic, $P \overline{1}$

$a=8.8647$ (4) ^

$b=9.4340(5) \AA$

$c=12.9119(6) \AA$

$\alpha=72.298$ (3)

$\beta=79.449$ (3)

\author{
Data collection \\ Bruker SMART APEX \\ diffractometer \\ Absorption correction: none \\ 8057 measured reflections \\ Refinement \\ $R\left[F^{2}>2 \sigma\left(F^{2}\right)\right]=0.061$ \\ $w R\left(F^{2}\right)=0.156$ \\ $S=0.97$ \\ 4343 reflections \\ 241 parameters \\ 15 restraints
}

4343 independent reflections 2454 reflections with $I>2 \sigma(I)$ $R_{\text {int }}=0.050$

Table 1

Hydrogen-bond geometry $\left(\AA{ }^{\circ}\right)$.

\begin{tabular}{lllll}
\hline$D-\mathrm{H} \cdots A$ & $D-\mathrm{H}$ & $\mathrm{H} \cdots A$ & $D \cdots A$ & $D-\mathrm{H} \cdots A$ \\
\hline $\mathrm{O} 1 w-\mathrm{H} 1 w 1 \cdots \mathrm{O} 2$ & $0.86(1)$ & $1.97(2)$ & $2.780(2)$ & $156(3)$ \\
$\mathrm{O} 1 w-\mathrm{H} 1 w 2 \cdots \mathrm{O} 4^{\mathrm{i}}$ & $0.86(1)$ & $1.97(2)$ & $2.780(2)$ & $157(3)$ \\
$\mathrm{N} 1-\mathrm{H} 1 n 1 \cdots \mathrm{O} 1$ & $0.85(1)$ & $1.94(1)$ & $2.788(2)$ & $172(2)$ \\
$\mathrm{N} 1-\mathrm{H} 1 n 2 \cdots \mathrm{O} 3^{\mathrm{ii}}$ & $0.87(1)$ & $1.91(1)$ & $2.755(2)$ & $167(2)$ \\
$\mathrm{N} 1-\mathrm{H} 1 n 3 \cdots \mathrm{O} 1 w^{\mathrm{iii}}$ & $0.86(1)$ & $1.99(1)$ & $2.823(3)$ & $164(2)$ \\
$\mathrm{N} 2-\mathrm{H} 2 n 1 \cdots \mathrm{O} 2$ & $0.86(1)$ & $2.35(2)$ & $2.996(2)$ & $132(2)$ \\
$\mathrm{N} 2-\mathrm{H} 2 n 1 \cdots \mathrm{O} 4^{\mathrm{i}}$ & $0.86(1)$ & $2.42(2)$ & $2.995(3)$ & $124(2)$ \\
$\mathrm{N} 2-\mathrm{H} 2 n 2 \cdots \mathrm{O} 2^{\mathrm{i}}$ & $0.87(1)$ & $1.91(1)$ & $2.781(2)$ & $172(2)$ \\
$\mathrm{N} 2-\mathrm{H} 2 n 3 \cdots \mathrm{O} 3$ & $0.87(1)$ & $1.89(1)$ & $2.741(2)$ & $166(2)$ \\
\hline Symmetry codes: (i) & $-x+2,-y+1,-z+1 ;(\mathrm{ii})$ & $-x+1,-y+1,-z+1 ;$ (iii) \\
$-x+1,-y+2,-z+1$. & &
\end{tabular}

Data collection: APEX2 (Bruker, 2007); cell refinement: SAINT (Bruker, 2007); data reduction: $S A I N T$; program(s) used to solve structure: SHELXS97 (Sheldrick, 2008); program(s) used to refine structure: SHELXL97 (Sheldrick, 2008); molecular graphics: $X$ $S E E D$ (Barbour, 2001); software used to prepare material for publication: publCIF (Westrip, 2008).

We acknowledge the SAGA grant (06-02-03-0147) for supporting this study, and the University of Malaya for the purchase of the diffractometer.

Supplementary data and figures for this paper are available from the IUCr electronic archives (Reference: LH2629).

\section{References}

Ariffin, A. \& Khan, M. N. (2005). Bull. Kor. Chem. Soc. 26, 1037-1043. Barbour, L. J. (2001). J. Supramol. Chem. 1, 189-191.

Bruker (2007). APEX2 and SAINT. Bruker AXS Inc., Madison, Wisconsin, USA.

Khan, M. N. \& Ariffin, A. (2003). Org. Biomol. Chem. 1, 1404-1408.

Sheldrick, G. M. (2008). Acta Cryst. A64, 112-122.

Westrip, S. P. (2008). publCIF. In preparation. 


\section{supporting information}

Acta Cryst. (2008). E64, o1152 [doi:10.1107/S1600536808015201]

\section{Bis(isobutylammonium) phthalate monohydrate}

\section{Zakaria Norzalida, Azhar Ariffin and Seik Weng Ng}

\section{S1. Comment}

The title salt (Fig. 1) was obtained as a wet crystalline compound when $N$-isobutylphthalimic acid was left aside for several years. The acid has been shown by kinetic studies to be converted to phthalic acid and isobutylamine under neutral and acidic conditions (Ariffin \& Khan, 2005; Khan Ariffin, 2003). In the anion, the carboxyl $-\mathrm{CO}_{2}$ groups are twisted with respect to the phenylene ring [dihedral angles 43.8 (1) and 50.9 (1) ${ }^{\circ}$. Hydrogen bonds which involve the ammonium cations and water molecules link the components of the salt into a layer motif (Table 1).

\section{S2. Experimental}

$N$-Isobutylphthalimidic acid was synthesized as described earlier (Ariffin \& Khan, 2005; Khan \& Ariffin, 2003). The crystalline compound was left aside for several years. The hydrolyzed title salt was obtained as a wet crystalline compound.

\section{S3. Refinement}

Carbon-bound H-atoms were placed in calculated positions ( $\mathrm{C}-\mathrm{H} 0.95$ to $0.99 \AA)$ and were included in the refinement in the riding model approximation, with $U(\mathrm{H})$ set to $1.2-1.5 U(\mathrm{C})$. The oxygen- and nitrogen-bound $\mathrm{H}$-atoms were located in a difference Fourier map, and were refined with restraints of $\mathrm{O}-\mathrm{H}=\mathrm{N}-\mathrm{H}=0.85 \pm 0.01 \AA ; \mathrm{H} \cdots \mathrm{H}=1.39 \pm 0.01 \AA$; their temperature factors were freely refined. 


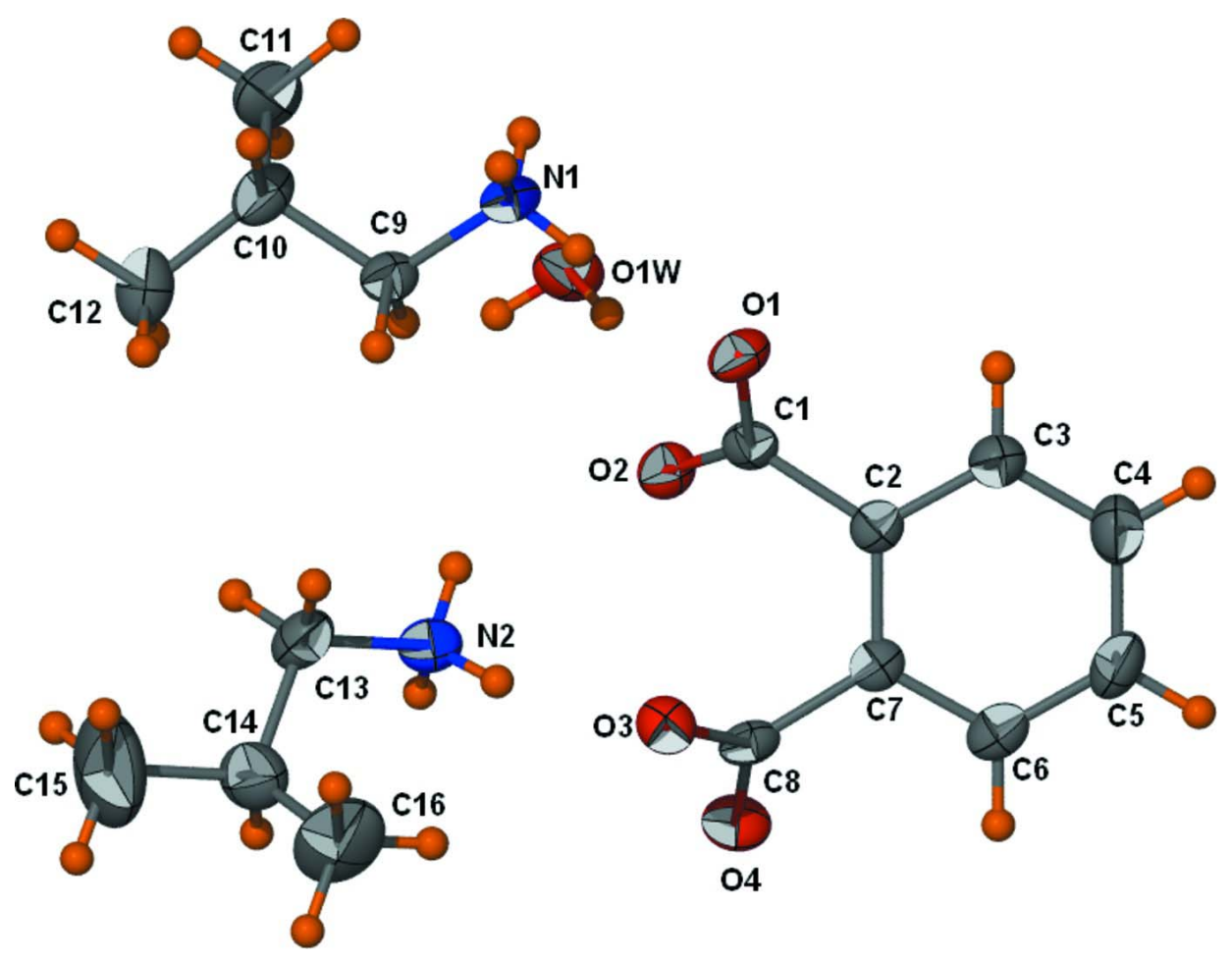

\section{Figure 1}

Thermal ellipsoid plot (Barbour, 2001) of $\left[\mathrm{C}_{4} \mathrm{H}_{12} \mathrm{~N}\right]_{2}\left[\mathrm{C}_{8} \mathrm{H}_{4} \mathrm{O}_{4}\right] \cdot \mathrm{H}_{2} \mathrm{O}$ at the $70 \%$ probability level. Hydrogen atoms are drawn as spheres of arbitrary radiius.

\section{Bis(isobutylammonium) phthalate monohydrate}

\section{Crystal data}

$2 \mathrm{C}_{4} \mathrm{H}_{12} \mathrm{~N}^{+} \cdot \mathrm{C}_{8} \mathrm{H}_{4} \mathrm{O}_{4}{ }^{2-} \cdot \mathrm{H}_{2} \mathrm{O}$

$M_{r}=330.42$

Triclinic, $P \overline{1}$

Hall symbol: -P 1

$a=8.8647(4) \AA$

$b=9.4340(5) \AA$

$c=12.9119(6) \AA$

$\alpha=72.298(3)^{\circ}$

$\beta=79.449(3)^{\circ}$

$\gamma=69.059(3)^{\circ}$

$V=957.37(8) \AA^{3}$

\section{Data collection}

Bruker SMART APEX diffractometer

Radiation source: fine-focus sealed tube

Graphite monochromator

$\omega$ scans

8057 measured reflections

4343 independent reflections
$Z=2$

$F(000)=360$

$D_{\mathrm{x}}=1.146 \mathrm{Mg} \mathrm{m}^{-3}$

Mo $K \alpha$ radiation, $\lambda=0.71073 \AA$

Cell parameters from 941 reflections

$\theta=2.5-22.7^{\circ}$

$\mu=0.08 \mathrm{~mm}^{-1}$

$T=100 \mathrm{~K}$

Prism, colorless

$0.32 \times 0.08 \times 0.08 \mathrm{~mm}$

2454 reflections with $I>2 \sigma(I)$

$R_{\text {int }}=0.050$

$\theta_{\text {max }}=27.5^{\circ}, \theta_{\text {min }}=2.5^{\circ}$

$h=-11 \rightarrow 11$

$k=-10 \rightarrow 12$

$l=-16 \rightarrow 16$ 


\section{Refinement}

Refinement on $F^{2}$

Least-squares matrix: full

$R\left[F^{2}>2 \sigma\left(F^{2}\right)\right]=0.061$

$w R\left(F^{2}\right)=0.156$

$S=0.97$

4343 reflections

241 parameters

15 restraints

Primary atom site location: structure-invariant direct methods

Secondary atom site location: difference Fourier map
Hydrogen site location: inferred from neighbouring sites

$\mathrm{H}$ atoms treated by a mixture of independent and constrained refinement

$w=1 /\left[\sigma^{2}\left(F_{\mathrm{o}}^{2}\right)+(0.0703 P)^{2}\right]$ where $P=\left(F_{\mathrm{o}}^{2}+2 F_{\mathrm{c}}{ }^{2}\right) / 3$

$(\Delta / \sigma)_{\max }=0.001$

$\Delta \rho_{\max }=0.33$ e $\AA^{-3}$

$\Delta \rho_{\text {min }}=-0.27$ e $\AA^{-3}$

Extinction correction: SHELXL97 (Sheldrick, 2008), $\mathrm{Fc}^{*}=\mathrm{kFc}\left[1+0.001 \times \mathrm{xc}^{2} \lambda^{3} / \sin (2 \theta)\right]^{-1 / 4}$

Extinction coefficient: 0.049 (5)

Fractional atomic coordinates and isotropic or equivalent isotropic displacement parameters $\left(\AA^{2}\right)$

\begin{tabular}{|c|c|c|c|c|}
\hline & $x$ & $y$ & $z$ & $U_{\text {iso }} * / U_{\text {eq }}$ \\
\hline $\mathrm{O} 1$ & 0.49468 (17) & 0.58257 (19) & $0.62388(12)$ & $0.0271(4)$ \\
\hline $\mathrm{O} 2$ & $0.75147(17)$ & $0.58034(18)$ & $0.58411(12)$ & $0.0250(4)$ \\
\hline $\mathrm{O} 3$ & $0.86510(16)$ & $0.23828(19)$ & $0.58460(12)$ & $0.0254(4)$ \\
\hline $\mathrm{O} 4$ & $1.08162(16)$ & $0.21636(19)$ & $0.66133(12)$ & $0.0285(4)$ \\
\hline O1w & $0.7147(2)$ & $0.8989(2)$ & $0.50641(15)$ & $0.0342(4)$ \\
\hline H1w1 & $0.696(3)$ & $0.811(2)$ & $0.535(2)$ & $0.078(12)^{*}$ \\
\hline H1w2 & $0.785(3)$ & $0.887(3)$ & $0.4520(18)$ & $0.096(14)^{*}$ \\
\hline N1 & $0.4142(2)$ & $0.8164(2)$ & $0.43079(16)$ & $0.0230(5)$ \\
\hline H1n1 & 0.448 & $0.7460(19)$ & $0.4886(14)$ & $0.062(10)^{*}$ \\
\hline $\mathrm{H} 1 \mathrm{n} 2$ & $0.3266(18)$ & $0.809(2)$ & $0.4159(16)$ & $0.034(7)^{*}$ \\
\hline $\mathrm{H} 1 \mathrm{n} 3$ & $0.391(2)$ & $0.9076(13)$ & $0.4421(17)$ & $0.028(7)^{*}$ \\
\hline N2 & $0.9312(2)$ & $0.4473(3)$ & $0.39672(16)$ & $0.0259(5)$ \\
\hline $\mathrm{H} 2 \mathrm{n} 1$ & $0.873(2)$ & $0.5321(15)$ & $0.4154(19)$ & $0.054(9)^{*}$ \\
\hline $\mathrm{H} 2 \mathrm{n} 2$ & $1.0323(12)$ & $0.440(2)$ & $0.3961(17)$ & $0.042(8)^{*}$ \\
\hline $\mathrm{H} 2 \mathrm{n} 3$ & $0.917(2)$ & $0.3689(16)$ & 0.4499 (14) & $0.037(8)^{*}$ \\
\hline $\mathrm{C} 1$ & $0.6391(3)$ & $0.5332(3)$ & $0.64401(17)$ & $0.0216(5)$ \\
\hline $\mathrm{C} 2$ & $0.6832(2)$ & $0.4119(3)$ & $0.75120(17)$ & $0.0212(5)$ \\
\hline $\mathrm{C} 3$ & $0.5823(3)$ & $0.4347(3)$ & $0.84480(18)$ & $0.0265(5)$ \\
\hline H3 & 0.4850 & 0.5215 & 0.8393 & $0.032 *$ \\
\hline $\mathrm{C} 4$ & $0.6217(3)$ & $0.3327(3)$ & $0.94606(19)$ & $0.0338(6)$ \\
\hline $\mathrm{H} 4$ & 0.5509 & 0.3489 & 1.0092 & $0.041 *$ \\
\hline $\mathrm{C} 5$ & $0.7644(3)$ & 0.2070 & $0.95501(19)$ & $0.0326(6)$ \\
\hline H5 & 0.7928 & 0.1378 & 1.0244 & $0.039 *$ \\
\hline C6 & $0.8653(3)$ & 0.1828 & $0.86233(18)$ & $0.0268(6)$ \\
\hline H6 & 0.9630 & 0.0964 & 0.8688 & $0.032 *$ \\
\hline $\mathrm{C} 7$ & $0.8264(2)$ & 0.2824 & $0.76022(17)$ & $0.0207(5)$ \\
\hline $\mathrm{C} 8$ & $0.9328(2)$ & $0.2442(3)$ & $0.66050(18)$ & $0.0217(5)$ \\
\hline C9 & $0.5386(2)$ & $0.7900(3)$ & $0.33858(17)$ & $0.0231(5)$ \\
\hline H9A & 0.5535 & 0.6865 & 0.3273 & $0.028^{*}$ \\
\hline H9B & 0.6430 & 0.7861 & 0.3584 & $0.028^{*}$ \\
\hline $\mathrm{C} 10$ & $0.4971(3)$ & $0.9152(3)$ & $0.23207(19)$ & $0.0309(6)$ \\
\hline $\mathrm{H} 10$ & 0.3883 & 0.9236 & 0.2148 & $0.037 *$ \\
\hline
\end{tabular}




\begin{tabular}{lllll} 
C11 & $0.4909(3)$ & $1.0755(3)$ & $0.2390(2)$ & $0.0464(8)$ \\
H11A & 0.4091 & 1.1074 & 0.2970 & $0.070^{*}$ \\
H11B & 0.4626 & 1.1529 & 0.1692 & $0.070^{*}$ \\
H11C & 0.5972 & 1.0693 & 0.2553 & $0.070^{*}$ \\
C12 & $0.6223(3)$ & $0.8638(3)$ & $0.14157(19)$ & $0.0369(7)$ \\
H12A & 0.6251 & 0.7605 & 0.1383 & $0.055^{*}$ \\
H12B & 0.7293 & 0.8575 & 0.1565 & $0.055^{*}$ \\
H12C & 0.5931 & 0.9405 & 0.0716 & $0.055^{*}$ \\
C13 & $0.8993(3)$ & $0.4525(3)$ & $0.28710(19)$ & $0.0308(6)$ \\
H13A & 0.9174 & 0.5469 & 0.2340 & $0.037^{*}$ \\
H13B & 0.7845 & 0.4611 & 0.2877 & $0.037^{*}$ \\
C14 & $1.0072(3)$ & $0.3079(3)$ & $0.2514(2)$ & $0.0397(7)$ \\
H14 & 1.1214 & 0.2921 & 0.2627 & $0.048^{*}$ \\
C15 & $0.9981(5)$ & $0.3334(5)$ & $0.1306(3)$ & $0.0870(13)$ \\
H15A & 1.0264 & 0.4277 & 0.0893 & $0.130^{*}$ \\
H15B & 0.8878 & 0.3469 & 0.1174 & $0.130^{*}$ \\
H15C & 1.0743 & 0.2421 & 0.1069 & $0.130^{*}$ \\
C16 & $0.9674(5)$ & $0.1631(4)$ & $0.3167(3)$ & $0.0663(10)$ \\
H16A & 0.9732 & 0.1493 & 0.3944 & $0.100^{*}$ \\
H16B & 1.0452 & 0.0715 & 0.2941 & $0.100^{*}$ \\
H16C & 0.8576 & 0.1736 & 0.3043 & $0.100^{*}$ \\
\hline
\end{tabular}

Atomic displacement parameters $\left(\AA^{2}\right)$

\begin{tabular}{lllllll}
\hline & $U^{11}$ & $U^{22}$ & $U^{33}$ & $U^{12}$ & $U^{13}$ & $U^{23}$ \\
\hline O1 & $0.0211(8)$ & $0.0247(10)$ & $0.0298(9)$ & $-0.0054(7)$ & $-0.0062(7)$ & $0.0010(7)$ \\
O2 & $0.0246(8)$ & $0.0226(9)$ & $0.0271(8)$ & $-0.0104(7)$ & $-0.0012(7)$ & $-0.0027(7)$ \\
O3 & $0.0224(8)$ & $0.0283(10)$ & $0.0282(9)$ & $-0.0094(7)$ & $-0.0021(7)$ & $-0.0094(7)$ \\
O4 & $0.0175(8)$ & $0.0308(10)$ & $0.0379(10)$ & $-0.0074(7)$ & $-0.0028(7)$ & $-0.0102(8)$ \\
O1w & $0.0366(10)$ & $0.0226(11)$ & $0.0420(11)$ & $-0.0109(8)$ & $0.0042(9)$ & $-0.0093(9)$ \\
N1 & $0.0209(10)$ & $0.0173(12)$ & $0.0286(12)$ & $-0.0047(8)$ & $-0.0045(9)$ & $-0.0031(10)$ \\
N2 & $0.0240(10)$ & $0.0219(12)$ & $0.0325(12)$ & $-0.0080(9)$ & $-0.0040(9)$ & $-0.0063(10)$ \\
C1 & $0.0250(11)$ & $0.0155(12)$ & $0.0251(12)$ & $-0.0066(10)$ & $-0.0013(10)$ & $-0.0068(10)$ \\
C2 & $0.0227(11)$ & $0.0195(13)$ & $0.0227(12)$ & $-0.0086(10)$ & $-0.0027(9)$ & $-0.0047(10)$ \\
C3 & $0.0275(12)$ & $0.0233(14)$ & $0.0256(13)$ & $-0.0045(10)$ & $-0.0033(10)$ & $-0.0060(10)$ \\
C4 & $0.0405(14)$ & $0.0363(17)$ & $0.0227(13)$ & $-0.0132(12)$ & $0.0010(11)$ & $-0.0059(12)$ \\
C5 & $0.0423(14)$ & $0.0301(15)$ & $0.0227(13)$ & $-0.0113(12)$ & $-0.0097(11)$ & $0.0005(11)$ \\
C6 & $0.0286(12)$ & $0.0230(14)$ & $0.0292(13)$ & $-0.0081(10)$ & $-0.0092(10)$ & $-0.0034(11)$ \\
C7 & $0.0203(11)$ & $0.0199(13)$ & $0.0235(12)$ & $-0.0093(10)$ & $-0.0032(9)$ & $-0.0039(10)$ \\
C8 & $0.0200(11)$ & $0.0136(12)$ & $0.0295(13)$ & $-0.0051(9)$ & $-0.0045(9)$ & $-0.0015(10)$ \\
C9 & $0.0207(11)$ & $0.0201(13)$ & $0.0270(12)$ & $-0.0065(9)$ & $-0.0023(9)$ & $-0.0038(10)$ \\
C10 & $0.0253(12)$ & $0.0320(15)$ & $0.0301(13)$ & $-0.0098(11)$ & $-0.0068(10)$ & $0.0027(11)$ \\
C11 & $0.0577(17)$ & $0.0253(16)$ & $0.0409(16)$ & $-0.0109(13)$ & $0.0083(14)$ & $0.0027(13)$ \\
C12 & $0.0443(15)$ & $0.0433(18)$ & $0.0267(13)$ & $-0.0213(13)$ & $-0.0027(12)$ & $-0.0059(12)$ \\
C13 & $0.0396(14)$ & $0.0233(14)$ & $0.0286(13)$ & $-0.0081(11)$ & $-0.0115(11)$ & $-0.0029(11)$ \\
C14 & $0.0430(15)$ & $0.0404(18)$ & $0.0359(15)$ & $-0.0071(13)$ & $-0.0066(12)$ & $-0.0156(13)$ \\
C15 & $0.139(4)$ & $0.091(3)$ & $0.0396(19)$ & $-0.038(3)$ & $-0.001(2)$ & $-0.031(2)$ \\
C16 & $0.116(3)$ & $0.0258(18)$ & $0.060(2)$ & $-0.0150(18)$ & $-0.023(2)$ & $-0.0156(16)$ \\
& & & & & & \\
\hline
\end{tabular}


Geometric parameters $\left(\AA,{ }^{\circ}\right)$

\begin{tabular}{|c|c|c|c|}
\hline $\mathrm{O} 1-\mathrm{C} 1$ & $1.243(2)$ & $\mathrm{C} 7-\mathrm{C} 8$ & $1.507(3)$ \\
\hline $\mathrm{O} 2-\mathrm{C} 1$ & $1.270(2)$ & $\mathrm{C} 9-\mathrm{C} 10$ & $1.519(3)$ \\
\hline $\mathrm{O} 3-\mathrm{C} 8$ & $1.263(3)$ & C9-H9A & 0.9900 \\
\hline $\mathrm{O} 4-\mathrm{C} 8$ & $1.251(2)$ & C9-H9B & 0.9900 \\
\hline $\mathrm{O} 1 \mathrm{w}-\mathrm{H} 1 \mathrm{w} 1$ & $0.861(10)$ & $\mathrm{C} 10-\mathrm{C} 12$ & $1.522(3)$ \\
\hline $\mathrm{O} 1 \mathrm{w}-\mathrm{H} 1 \mathrm{w} 2$ & $0.858(10)$ & $\mathrm{C} 10-\mathrm{C} 11$ & $1.523(4)$ \\
\hline $\mathrm{N} 1-\mathrm{C} 9$ & $1.483(3)$ & $\mathrm{C} 10-\mathrm{H} 10$ & 1.0000 \\
\hline $\mathrm{N} 1-\mathrm{H} 1 \mathrm{n} 1$ & $0.852(9)$ & $\mathrm{C} 11-\mathrm{H} 11 \mathrm{~A}$ & 0.9800 \\
\hline $\mathrm{N} 1-\mathrm{H} 1 \mathrm{n} 2$ & $0.865(9)$ & $\mathrm{C} 11-\mathrm{H} 11 \mathrm{~B}$ & 0.9800 \\
\hline $\mathrm{N} 1-\mathrm{H} 1 \mathrm{n} 3$ & $0.861(9)$ & $\mathrm{C} 11-\mathrm{H} 11 \mathrm{C}$ & 0.9800 \\
\hline $\mathrm{N} 2-\mathrm{C} 13$ & $1.477(3)$ & $\mathrm{C} 12-\mathrm{H} 12 \mathrm{~A}$ & 0.9800 \\
\hline $\mathrm{N} 2-\mathrm{H} 2 \mathrm{n} 1$ & $0.860(9)$ & $\mathrm{C} 12-\mathrm{H} 12 \mathrm{~B}$ & 0.9800 \\
\hline $\mathrm{N} 2-\mathrm{H} 2 \mathrm{n} 2$ & $0.874(9)$ & $\mathrm{C} 12-\mathrm{H} 12 \mathrm{C}$ & 0.9800 \\
\hline $\mathrm{N} 2-\mathrm{H} 2 \mathrm{n} 3$ & $0.873(9)$ & $\mathrm{C} 13-\mathrm{C} 14$ & $1.508(4)$ \\
\hline $\mathrm{C} 1-\mathrm{C} 2$ & $1.514(3)$ & $\mathrm{C} 13-\mathrm{H} 13 \mathrm{~A}$ & 0.9900 \\
\hline $\mathrm{C} 2-\mathrm{C} 3$ & $1.389(3)$ & $\mathrm{C} 13-\mathrm{H} 13 \mathrm{~B}$ & 0.9900 \\
\hline $\mathrm{C} 2-\mathrm{C} 7$ & $1.406(3)$ & $\mathrm{C} 14-\mathrm{C} 16$ & $1.496(4)$ \\
\hline $\mathrm{C} 3-\mathrm{C} 4$ & $1.386(3)$ & $\mathrm{C} 14-\mathrm{C} 15$ & $1.518(4)$ \\
\hline $\mathrm{C} 3-\mathrm{H} 3$ & 0.9500 & $\mathrm{C} 14-\mathrm{H} 14$ & 1.0000 \\
\hline $\mathrm{C} 4-\mathrm{C} 5$ & $1.384(3)$ & $\mathrm{C} 15-\mathrm{H} 15 \mathrm{~A}$ & 0.9800 \\
\hline $\mathrm{C} 4-\mathrm{H} 4$ & 0.9500 & $\mathrm{C} 15-\mathrm{H} 15 \mathrm{~B}$ & 0.9800 \\
\hline $\mathrm{C} 5-\mathrm{C} 6$ & $1.383(3)$ & $\mathrm{C} 15-\mathrm{H} 15 \mathrm{C}$ & 0.9800 \\
\hline $\mathrm{C} 5-\mathrm{H} 5$ & 0.9500 & $\mathrm{C} 16-\mathrm{H} 16 \mathrm{~A}$ & 0.9800 \\
\hline $\mathrm{C} 6-\mathrm{C} 7$ & $1.386(3)$ & $\mathrm{C} 16-\mathrm{H} 16 \mathrm{~B}$ & 0.9800 \\
\hline $\mathrm{C} 6-\mathrm{H} 6$ & 0.9500 & $\mathrm{C} 16-\mathrm{H} 16 \mathrm{C}$ & 0.9800 \\
\hline $\mathrm{H} 1 \mathrm{w} 1-\mathrm{O} 1 \mathrm{w}-\mathrm{H} 1 \mathrm{w} 2$ & $107.2(15)$ & $\mathrm{C} 9-\mathrm{C} 10-\mathrm{C} 12$ & $108.7(2)$ \\
\hline $\mathrm{C} 9-\mathrm{N} 1-\mathrm{H} 1 \mathrm{n} 1$ & $109.2(17)$ & $\mathrm{C} 9-\mathrm{C} 10-\mathrm{C} 11$ & $112.0(2)$ \\
\hline $\mathrm{C} 9-\mathrm{N} 1-\mathrm{H} 1 \mathrm{n} 2$ & $108.1(14)$ & $\mathrm{C} 12-\mathrm{C} 10-\mathrm{C} 11$ & $110.4(2)$ \\
\hline $\mathrm{H} 1 \mathrm{n} 1-\mathrm{N} 1-\mathrm{H} 1 \mathrm{n} 2$ & $109.4(13)$ & $\mathrm{C} 9-\mathrm{C} 10-\mathrm{H} 10$ & 108.5 \\
\hline $\mathrm{C} 9-\mathrm{N} 1-\mathrm{H} 1 \mathrm{n} 3$ & $113.4(15)$ & $\mathrm{C} 12-\mathrm{C} 10-\mathrm{H} 10$ & 108.5 \\
\hline $\mathrm{H} 1 \mathrm{n} 1-\mathrm{N} 1-\mathrm{H} 1 \mathrm{n} 3$ & $108.9(13)$ & $\mathrm{C} 11-\mathrm{C} 10-\mathrm{H} 10$ & 108.5 \\
\hline $\mathrm{H} 1 \mathrm{n} 2-\mathrm{N} 1-\mathrm{H} 1 \mathrm{n} 3$ & $107.9(12)$ & $\mathrm{C} 10-\mathrm{C} 11-\mathrm{H} 11 \mathrm{~A}$ & 109.5 \\
\hline $\mathrm{C} 13-\mathrm{N} 2-\mathrm{H} 2 \mathrm{n} 1$ & $111.6(16)$ & $\mathrm{C} 10-\mathrm{C} 11-\mathrm{H} 11 \mathrm{~B}$ & 109.5 \\
\hline $\mathrm{C} 13-\mathrm{N} 2-\mathrm{H} 2 \mathrm{n} 2$ & $109.3(15)$ & $\mathrm{H} 11 \mathrm{~A}-\mathrm{C} 11-\mathrm{H} 11 \mathrm{~B}$ & 109.5 \\
\hline $\mathrm{H} 2 \mathrm{n} 1-\mathrm{N} 2-\mathrm{H} 2 \mathrm{n} 2$ & $106.4(13)$ & $\mathrm{C} 10-\mathrm{C} 11-\mathrm{H} 11 \mathrm{C}$ & 109.5 \\
\hline $\mathrm{C} 13-\mathrm{N} 2-\mathrm{H} 2 \mathrm{n} 3$ & $116.3(15)$ & $\mathrm{H} 11 \mathrm{~A}-\mathrm{C} 11-\mathrm{H} 11 \mathrm{C}$ & 109.5 \\
\hline $\mathrm{H} 2 \mathrm{n} 1-\mathrm{N} 2-\mathrm{H} 2 \mathrm{n} 3$ & $107.0(13)$ & $\mathrm{H} 11 \mathrm{~B}-\mathrm{C} 11-\mathrm{H} 11 \mathrm{C}$ & 109.5 \\
\hline $\mathrm{H} 2 \mathrm{n} 2-\mathrm{N} 2-\mathrm{H} 2 \mathrm{n} 3$ & $105.7(13)$ & $\mathrm{C} 10-\mathrm{C} 12-\mathrm{H} 12 \mathrm{~A}$ & 109.5 \\
\hline $\mathrm{O} 1-\mathrm{C} 1-\mathrm{O} 2$ & $125.4(2)$ & $\mathrm{C} 10-\mathrm{C} 12-\mathrm{H} 12 \mathrm{~B}$ & 109.5 \\
\hline $\mathrm{O} 1-\mathrm{C} 1-\mathrm{C} 2$ & $117.22(19)$ & $\mathrm{H} 12 \mathrm{~A}-\mathrm{C} 12-\mathrm{H} 12 \mathrm{~B}$ & 109.5 \\
\hline $\mathrm{O} 2-\mathrm{C} 1-\mathrm{C} 2$ & $117.30(18)$ & $\mathrm{C} 10-\mathrm{C} 12-\mathrm{H} 12 \mathrm{C}$ & 109.5 \\
\hline $\mathrm{C} 3-\mathrm{C} 2-\mathrm{C} 7$ & $119.0(2)$ & $\mathrm{H} 12 \mathrm{~A}-\mathrm{C} 12-\mathrm{H} 12 \mathrm{C}$ & 109.5 \\
\hline $\mathrm{C} 3-\mathrm{C} 2-\mathrm{C} 1$ & $118.49(19)$ & $\mathrm{H} 12 \mathrm{~B}-\mathrm{C} 12-\mathrm{H} 12 \mathrm{C}$ & 109.5 \\
\hline $\mathrm{C} 7-\mathrm{C} 2-\mathrm{C} 1$ & $122.38(19)$ & $\mathrm{N} 2-\mathrm{C} 13-\mathrm{C} 14$ & $111.78(19)$ \\
\hline $\mathrm{C} 4-\mathrm{C} 3-\mathrm{C} 2$ & $121.0(2)$ & $\mathrm{N} 2-\mathrm{C} 13-\mathrm{H} 13 \mathrm{~A}$ & 109.3 \\
\hline
\end{tabular}




\begin{tabular}{|c|c|c|c|}
\hline $\mathrm{C} 4-\mathrm{C} 3-\mathrm{H} 3$ & 119.5 & $\mathrm{C} 14-\mathrm{C} 13-\mathrm{H} 13 \mathrm{~A}$ & 109.3 \\
\hline $\mathrm{C} 2-\mathrm{C} 3-\mathrm{H} 3$ & 119.5 & $\mathrm{~N} 2-\mathrm{C} 13-\mathrm{H} 13 \mathrm{~B}$ & 109.3 \\
\hline $\mathrm{C} 5-\mathrm{C} 4-\mathrm{C} 3$ & $119.9(2)$ & $\mathrm{C} 14-\mathrm{C} 13-\mathrm{H} 13 \mathrm{~B}$ & 109.3 \\
\hline $\mathrm{C} 5-\mathrm{C} 4-\mathrm{H} 4$ & 120.1 & $\mathrm{H} 13 \mathrm{~A}-\mathrm{C} 13-\mathrm{H} 13 \mathrm{~B}$ & 107.9 \\
\hline $\mathrm{C} 3-\mathrm{C} 4-\mathrm{H} 4$ & 120.1 & $\mathrm{C} 16-\mathrm{C} 14-\mathrm{C} 13$ & $112.4(2)$ \\
\hline $\mathrm{C} 6-\mathrm{C} 5-\mathrm{C} 4$ & $119.6(2)$ & $\mathrm{C} 16-\mathrm{C} 14-\mathrm{C} 15$ & $110.8(3)$ \\
\hline $\mathrm{C} 6-\mathrm{C} 5-\mathrm{H} 5$ & 120.2 & $\mathrm{C} 13-\mathrm{C} 14-\mathrm{C} 15$ & $110.0(3)$ \\
\hline $\mathrm{C} 4-\mathrm{C} 5-\mathrm{H} 5$ & 120.2 & $\mathrm{C} 16-\mathrm{C} 14-\mathrm{H} 14$ & 107.8 \\
\hline $\mathrm{C} 5-\mathrm{C} 6-\mathrm{C} 7$ & $121.2(2)$ & $\mathrm{C} 13-\mathrm{C} 14-\mathrm{H} 14$ & 107.8 \\
\hline $\mathrm{C} 5-\mathrm{C} 6-\mathrm{H} 6$ & 119.4 & $\mathrm{C} 15-\mathrm{C} 14-\mathrm{H} 14$ & 107.8 \\
\hline $\mathrm{C} 7-\mathrm{C} 6-\mathrm{H} 6$ & 119.4 & $\mathrm{C} 14-\mathrm{C} 15-\mathrm{H} 15 \mathrm{~A}$ & 109.5 \\
\hline $\mathrm{C} 6-\mathrm{C} 7-\mathrm{C} 2$ & $119.3(2)$ & $\mathrm{C} 14-\mathrm{C} 15-\mathrm{H} 15 \mathrm{~B}$ & 109.5 \\
\hline $\mathrm{C} 6-\mathrm{C} 7-\mathrm{C} 8$ & $119.4(2)$ & $\mathrm{H} 15 \mathrm{~A}-\mathrm{C} 15-\mathrm{H} 15 \mathrm{~B}$ & 109.5 \\
\hline $\mathrm{C} 2-\mathrm{C} 7-\mathrm{C} 8$ & $121.22(19)$ & $\mathrm{C} 14-\mathrm{C} 15-\mathrm{H} 15 \mathrm{C}$ & 109.5 \\
\hline $\mathrm{O} 4-\mathrm{C} 8-\mathrm{O} 3$ & $125.5(2)$ & $\mathrm{H} 15 \mathrm{~A}-\mathrm{C} 15-\mathrm{H} 15 \mathrm{C}$ & 109.5 \\
\hline $\mathrm{O} 4-\mathrm{C} 8-\mathrm{C} 7$ & $117.10(19)$ & $\mathrm{H} 15 \mathrm{~B}-\mathrm{C} 15-\mathrm{H} 15 \mathrm{C}$ & 109.5 \\
\hline $\mathrm{O} 3-\mathrm{C} 8-\mathrm{C} 7$ & $117.40(18)$ & $\mathrm{C} 14-\mathrm{C} 16-\mathrm{H} 16 \mathrm{~A}$ & 109.5 \\
\hline $\mathrm{N} 1-\mathrm{C} 9-\mathrm{C} 10$ & $113.95(18)$ & $\mathrm{C} 14-\mathrm{C} 16-\mathrm{H} 16 \mathrm{~B}$ & 109.5 \\
\hline $\mathrm{N} 1-\mathrm{C} 9-\mathrm{H} 9 \mathrm{~A}$ & 108.8 & $\mathrm{H} 16 \mathrm{~A}-\mathrm{C} 16-\mathrm{H} 16 \mathrm{~B}$ & 109.5 \\
\hline $\mathrm{C} 10-\mathrm{C} 9-\mathrm{H} 9 \mathrm{~A}$ & 108.8 & $\mathrm{C} 14-\mathrm{C} 16-\mathrm{H} 16 \mathrm{C}$ & 109.5 \\
\hline $\mathrm{N} 1-\mathrm{C} 9-\mathrm{H} 9 \mathrm{~B}$ & 108.8 & $\mathrm{H} 16 \mathrm{~A}-\mathrm{C} 16-\mathrm{H} 16 \mathrm{C}$ & 109.5 \\
\hline $\mathrm{C} 10-\mathrm{C} 9-\mathrm{H} 9 \mathrm{~B}$ & 108.8 & $\mathrm{H} 16 \mathrm{~B}-\mathrm{C} 16-\mathrm{H} 16 \mathrm{C}$ & 109.5 \\
\hline $\mathrm{H} 9 \mathrm{~A}-\mathrm{C} 9-\mathrm{H} 9 \mathrm{~B}$ & 107.7 & & \\
\hline $\mathrm{O} 1-\mathrm{C} 1-\mathrm{C} 2-\mathrm{C} 3$ & $-43.9(3)$ & $\mathrm{C} 1-\mathrm{C} 2-\mathrm{C} 7-\mathrm{C} 6$ & $174.98(19)$ \\
\hline $\mathrm{O} 2-\mathrm{C} 1-\mathrm{C} 2-\mathrm{C} 3$ & $133.5(2)$ & $\mathrm{C} 3-\mathrm{C} 2-\mathrm{C} 7-\mathrm{C} 8$ & $174.7(2)$ \\
\hline $\mathrm{O} 1-\mathrm{C} 1-\mathrm{C} 2-\mathrm{C} 7$ & $139.7(2)$ & $\mathrm{C} 1-\mathrm{C} 2-\mathrm{C} 7-\mathrm{C} 8$ & $-8.9(3)$ \\
\hline $\mathrm{O} 2-\mathrm{C} 1-\mathrm{C} 2-\mathrm{C} 7$ & $-42.9(3)$ & $\mathrm{C} 6-\mathrm{C} 7-\mathrm{C} 8-\mathrm{O} 4$ & $-51.4(3)$ \\
\hline $\mathrm{C} 7-\mathrm{C} 2-\mathrm{C} 3-\mathrm{C} 4$ & $0.4(3)$ & $\mathrm{C} 2-\mathrm{C} 7-\mathrm{C} 8-\mathrm{O} 4$ & $132.5(2)$ \\
\hline $\mathrm{C} 1-\mathrm{C} 2-\mathrm{C} 3-\mathrm{C} 4$ & $-176.1(2)$ & $\mathrm{C} 6-\mathrm{C} 7-\mathrm{C} 8-\mathrm{O} 3$ & $126.5(2)$ \\
\hline $\mathrm{C} 2-\mathrm{C} 3-\mathrm{C} 4-\mathrm{C} 5$ & $0.8(4)$ & $\mathrm{C} 2-\mathrm{C} 7-\mathrm{C} 8-\mathrm{O} 3$ & $-49.7(3)$ \\
\hline $\mathrm{C} 3-\mathrm{C} 4-\mathrm{C} 5-\mathrm{C} 6$ & $-1.1(4)$ & $\mathrm{N} 1-\mathrm{C} 9-\mathrm{C} 10-\mathrm{C} 12$ & $-172.76(19)$ \\
\hline $\mathrm{C} 4-\mathrm{C} 5-\mathrm{C} 6-\mathrm{C} 7$ & $0.1(4)$ & $\mathrm{N} 1-\mathrm{C} 9-\mathrm{C} 10-\mathrm{C} 11$ & $65.0(3)$ \\
\hline $\mathrm{C} 5-\mathrm{C} 6-\mathrm{C} 7-\mathrm{C} 2$ & $1.2(3)$ & $\mathrm{N} 2-\mathrm{C} 13-\mathrm{C} 14-\mathrm{C} 16$ & $68.6(3)$ \\
\hline $\mathrm{C} 5-\mathrm{C} 6-\mathrm{C} 7-\mathrm{C} 8$ & $-175.0(2)$ & $\mathrm{N} 2-\mathrm{C} 13-\mathrm{C} 14-\mathrm{C} 15$ & $-167.4(2)$ \\
\hline $\mathrm{C} 3-\mathrm{C} 2-\mathrm{C} 7-\mathrm{C} 6$ & $-1.4(3)$ & & \\
\hline
\end{tabular}

Hydrogen-bond geometry $\left(\AA,{ }^{\circ}\right)$

\begin{tabular}{lllll}
\hline$D-\mathrm{H} \cdots A$ & $D-\mathrm{H}$ & $\mathrm{H} \cdots A$ & $D \cdots A$ & $D-\mathrm{H}^{\cdots} A$ \\
\hline $\mathrm{O} 1 w-\mathrm{H} 1 w 1 \cdots \mathrm{O} 2$ & $0.86(1)$ & $1.97(2)$ & $2.780(2)$ & $156(3)$ \\
$\mathrm{O} 1 w-\mathrm{H} 1 w 2 \cdots \mathrm{O} 4^{\mathrm{i}}$ & $0.86(1)$ & $1.97(2)$ & $2.780(2)$ & $157(3)$ \\
$\mathrm{N} 1-\mathrm{H} 1 n 1 \cdots \mathrm{O} 1$ & $0.85(1)$ & $1.94(1)$ & $2.788(2)$ & $172(2)$ \\
$\mathrm{N} 1-\mathrm{H} 1 n 2 \cdots \mathrm{O} 3^{\mathrm{ii}}$ & $0.87(1)$ & $1.91(1)$ & $2.755(2)$ & $167(2)$ \\
$\mathrm{N} 1-\mathrm{H} 1 n 3 \cdots \mathrm{O} 1 w^{\text {iii }}$ & $0.86(1)$ & $1.99(1)$ & $2.823(3)$ & $164(2)$ \\
$\mathrm{N} 2-\mathrm{H} 2 n 1 \cdots \mathrm{O} 2$ & $0.86(1)$ & $2.35(2)$ & $2.996(2)$ & $132(2)$ \\
$\mathrm{N} 2-\mathrm{H} 2 n 1 \cdots \mathrm{O} 4{ }^{\mathrm{i}}$ & $0.86(1)$ & $2.42(2)$ & $2.995(3)$ & $124(2)$
\end{tabular}


supporting information

$\mathrm{N} 2-\mathrm{H} 2 n 2 \cdots \mathrm{O} 2$

$0.87(1)$

$1.91(1)$

$2.781(2)$

$172(2)$

$\mathrm{N} 2-\mathrm{H} 2 n 3 \cdots \mathrm{O} 3$

$0.87(1)$

1.89 (1)

$2.741(2)$

$166(2)$

Symmetry codes: (i) $-x+2,-y+1,-z+1$; (ii) $-x+1,-y+1,-z+1$; (iii) $-x+1,-y+2,-z+1$. 